\title{
Mesoporous silica Nanoparticles as Drug Delivery System
}

\section{Dennis Douroumis}

University of Greenwich, School of Sciences Medway Campus, Chatham Maritime, Kent, UK

The last ten years mesoporous silica nanoparticles(MSNs) have been extensive investigated as a drug delivery system. It is well know that MSNs possess excellent properties such high specific area, high pore volume, tunable pore structures and physicochemical stability. In the beginning MSNs were used for controlled delivery of various hydrophilic or hydrophobic active agents. Later advances in the MSNs surface properties such as surface functionalization and PEGylation rendered them as a promising drug delivery vehicle for cancer treatment.

However, there are major concerns about the biocompatibility, toxicity, in vivo biodistribution and efficacy of MSNs of various particle sizes. Another drawback is the premature drug leakage from the silica pores resulting reduced delivery of the actives at the desired sites. These properties play a key role for the successful utilization of MSNs and further experimental findings will assist in the design of effective drug delivery systems.

Recent studies revealed interesting results regarding the aforementioned properties and also the drug delivered to the cancer sites. In vitro evaluation of anticancer agents such as camptothecin and irinotecan hydrochloride trihydrate in pancreatic (PANC-1, Capan-1, and AsPc-1) [1] and breast MCF-7 cancer-cell lines showed controlled release of the actives, size dependent cellular uptake and remarkable anticancer efficacy. Interestingly, in vivo animal studies demonstrated contradictory results. When MSNs of various sizes were administrated subcutaneously to mice [3] appeared to be non toxic and the amount of residual material decreased progressively over 3 months, with good biocompatibility on histology at all time points. The same doses of MSNs administrated intra-peritoneal and intra-venous injections in mice resulted in death or euthanasia. In contrast, other studies [4] demonstrated that fluorescent MSNs are biocompatible at effective dosages, able of reducing toxicity of anticancer drugs, accumulate in tumour xenografts with or without targeting moieties and exhibit excellent tumour suppressing effect.
More recently, PEGylated MSNs presented low systemic toxicity in healthy mice and enhanced tumour inhibition rate on marine hepatocarcinoma 22 models [5]. The in vivo biodistribution and urinary excretion of spherical PEGylated MSNs [6] showed liver and spleen distribution with a minority of particles in the lungs and even fewer in the kidney or heart. PEGylated MSNs of smaller particle sizes possess longer blood-circulation lifetime, slow biodegradation times and lower excreted amount of degradation products in the urine.

In spite of the considerable interest in the biomedical applications of MSNs the knowledge on the in vivo biocompatibility, toxicity and in vivo biodistribution of MSNs is still very limited. Most importantly there is a lack of human clinical trials and the few existing studies in animal models cannot provide sufficient evidence on MSNs safety. Nevertheless, these preliminary in vivo trials suggest that MSNs are a promising drug delivery system but still significant improvements and clinical studies are required.

\section{References}

1. Lu J, Liong M, Zink JI, Tamanoi F (2007) Mesoporous silica nanoparticles as a delivery system for hydrophobic anticancer drugs. Small 3: 1341-6.

2. He Q, Shi J, Chen F, Zhu M, Zhang L (2008) An anticancer drug delivery system based on surfactant-templated mesoporous silica nanoparticles. Biomaterials 2010 31:3335-3346.

3. Hudson SP, Padera RF, Langer R, Kohane DS (2008) The biocompatibility of mesoporous silicates. Biomaterials. 29: 4045-55.

4. Lu J, Liong M, Li Z, Zink JI, Tamanoi F (2010) Biocompatibility, biodistribution and drug-delivery efficiency of mesoporous silica nanoparticles for cancer therapy in animals. Small 6: 1794-1805.

5. He Q, Zhang Z, Gao F, Li Y, Shi J (2011) In vivo biodistribution and urinary excretion of mesoporous silica nanoparticles: effects of particle size and PEGylation. Small 7: 271-280.

6. Li L, Tang F, Liu H, Liu T, Hao N, et al. (2010) In vivo delivery of silica nanorattle encapsulated docetaxel for liver cancer therapy with low toxicity and high efficacy. ACS Nano 4: 6874-6882.
Corresponding author: Dennis Douroumis, University of Greenwich, School of Sciences Medway Campus, Chatham Maritime, Kent, UK, E-mail: d.douroumis@ gre.ac.uk

Received May 24, 2011; Accepted May 25, 2011; Published May 26, 2011

Citation: Douroumis D (2011) Mesoporous silica Nanoparticles as Drug Delivery System. J Nanomedic Nanotechnol 2:102e. doi:10.4172/2157-7439.1000102e

Copyright: ( 2011 Douroumis D. This is an open-access article distributed under the terms of the Creative Commons Attribution License, which permits unrestricted use, distribution, and reproduction in any medium, provided the original author and source are credited. 